\title{
Clinical outcome of advanced squamous cell carcinoma of the conjunctiva
}

\author{
Abstract \\ Background To evaluate the outcome of \\ surgical management of advanced squamous \\ cell carcinoma (SCC) of the conjunctiva \\ (American Joint Committee Cancer- \\ classification $>$ III) and the rate of \\ recurrences after treatment during follow-up. \\ Second, to investigate the incidence of orbital \\ exenteration during follow-up. \\ Methods Thirty-eight cases with SCC \\ $>$ grade T3 AJCC were retrospectively \\ analysed at a University Eye Hospital \\ Munich. Tumour stage, type of treatment, \\ follow-up time, risk factors and-if present- \\ recurrence were documented. \\ Results The mean follow-up was $\mathbf{2 4 . 2}$ \\ months (22.3-71 months). The most frequent \\ surgical procedure was local tumour excision \\ ( $n=25$ patients, $71 \%$ ). Orbital exenteration \\ was performed in 10 patients $(28 \%)$. Twenty \\ patients $(57 \%)$ did not show a progressive \\ disease during follow-up. Of the patients \\ with primary local excision, $13(52 \%)$ had \\ recurrence. Average time to recurrence for all \\ treated patients was 24 months in the mean \\ (minimum 4 months, maximum 68 months, \\ SD, 22). Patients following orbital \\ exenteration had recurrence of disease in $20 \%$ \\ $(n=2)$. None of the patients with primarily \\ local tumour excision required an orbital \\ exenteration. \\ Conclusion Advanced-stage SCC can be \\ treated surgically. An extensive surgical \\ approach is sometimes inevitable. Patients with \\ surgical excision of advanced-stage disease \\ should be reviewed closely as recurrences may \\ occur and even after more than 5 years. \\ However, on early detection, most of these \\ recurrences can be handled by local excision. \\ Eye (2014) 28, 962-967; doi:10.1038/eye.2014.79; \\ published online 23 May 2014
}

Department of Ophthalmology, Ludwig-MaximiliansUniversität, München, Germany

Correspondence: CV Miller, Department of Ophthalmology, Ludwig-MaximiliansUniversität, Mathildenstr. 8, 80336 München, Germany Tel: + 498951603811 ; Fax: +498951605160. E-mail: christina.miller@ med.uni-muenchen.de

Received: 10 November 2013

Accepted in revised form: 26 February 2014 Published online: 23 May 2014
CV Miller, A Wolf, A Klingenstein, C Decker, A Garip, A Kampik and C Hintschich

\section{Introduction}

Squamous cell carcinoma (SCC) of the conjunctiva is a rare malignancy; however, it is reported to be the most common malignant tumour of the ocular surface. Its incidence varies between 0.02 and 3.5/100.000. ${ }^{1}$ In SCC the abnormal cells extend through the basement membrane into the conjunctival stroma, whereas in intraepithelial neoplasia (CIN) the malignant cells are confined to the surface epithelium. SCC has the potential to penetrate the corneoscleral lamella into the anterior chamber of the eye or can breach the orbital septum to invade the soft tissues of the orbit, sinuses, and the brain. ${ }^{2,3}$ These tumours may metastasize via lymphatics or blood during the course of disease. ${ }^{3}$ Owing to their possible aggressive behaviour, conjunctival SCCs are therefore known to be sight and life threatening. ${ }^{3,4}$ Although there are different treatment modalities available, surgical excision with or without cryotherapy remains the widely accepted treatment strategy for SCC of the conjunctiva. 3,5,6 Other treatment modalities currently in use are radiotherapy, ${ }^{7}$ surgical excision in combination with absolute alcohol, ${ }^{8}$ vitamin $\mathrm{A},{ }^{9}$ excimer laser, ${ }^{10}$ and the use of adjuvant topical or perilesional chemotherapy. ${ }^{11-18}$ Recent groups focus on the off-label use of interferon $\alpha-2 b$ (IFN $\alpha-2 b)$, especially on epithelial dysplasia and lowgrade epithelial malignancies of the conjunctiva. $16,17,19,20$ Some groups report favourable outcomes for advanced-stage disease using topical or subconjunctival IFN $\alpha$-2b. ${ }^{18}$ However, the published data on recurrences after surgical excision of advanced tumours show a large variation between 5 and $56 \% .^{21,22}$ 
The purpose of this study was to evaluate the outcome of surgical management of advanced SCC of the conjunctiva (American Joint Committee Cancer (AJCC) classification $>$ III) and the rate of recurrences after treatment during follow-up. The other purpose of this study was to investigate the necessity of primary orbital exenteration.

\section{Patients and methods}

The study was planned as a retrospective study and IRB approval was obtained in January 2011.

\section{Patients}

Medical records of patients treated between January 2005 and April 2012 at the University Eye Hospital at Ludwigs-Maximilians-Universität (LMU) Munich were analysed.

Inclusion criteria were histopathologically proven advanced SCCs of the conjunctiva (> grade III AJCC classification). Patients with histologically evident squamous cell dysplasia or SCC in situ were not included into the study.

Exclusion criterion was previous treatment of SCC in our hospital.

Main criterion of the study was the outcome of surgical treatment, secondary outcomes were the incidence of orbital exenteration during our follow-up period and the disease-related mortality.

Overall, 38 patients were identified. All patients were treated at the University Eye Hospital Munich (LMU). An incisional biopsy of the conjunctival lesion was performed in all patients to prove the diagnosis of SCC histologically. For all patients, demographic data as well as known risk factors for SCC (cigarette smoking, existing atopic diseases, immunosuppressive medication, and human immunodeficiency virus infection) were collected. In case of recurrence, time span between last surgery and recurrence was documented. The clinical findings of every tumour at first presentation in our clinic were recorded before treatment. Slitlamp biomicroscopy and clinical photographs were taken at every visit of the primary site of involvement and the status of regional preauricular and submandibular lymph nodes were assessed each time. For all cases, growth pattern (diffuse/nodular) and involvement of adjacent structures (cornea, forniceal conjunctiva, palpebral conjunctiva, tarsal conjunctiva, intraocular compartments, caruncle, lacrimal punctum, canaliculi, semilunar fold, anterior or posterior eyelid lamellae, and/ or eyelid margin) were documented. If tumour invasion into the orbit was suspected, a contrast enhanced computed tomography scan of the orbits was obtained. On the basis of these findings, each tumour was graded according to the AJCC classification (Table 1) at first presentation. ${ }^{23}$ The term 'advanced SCC' was defined as tumours in stage T3 and T4 based on the AJCC classification. ${ }^{20}$

\section{Treatment}

Surgical excision of all cases was carried out by a single ophthalmic surgeon $(\mathrm{CH})$. A 2-mm safety margin was used as a standard of care in tumour surgery. However, if complete tumour excision was not feasible due to the site or the size of the lesion, an adjuvant chemotherapy with topical IFN $\alpha-2 b$ was additionally used. IFN $\alpha-2 b$, available as Intron A (Essex Pharma, Luzern, Switzerland), was diluted under sterile conditions with distilled sterile water to one million international units per $\mathrm{ml}$ and packed in single-dose units (EDO) by a local pharmacy. It was refrigerated (at $4{ }^{\circ} \mathrm{C}$ ) and applied one drop $5 \times$ per day for 6 weeks.

If the tumour invaded deep orbital structures, which were not accessible for local excision, an orbital exenteration was carried out.

\section{Data collection}

All data were collected in a MS-Excel 2000 spreadsheet (Microsoft Corporation, Unterschleissheim, Germany) and statistical analysis was performed using SPSS 19.0 for Windows (SPSS Inc., IBM Company, Chicago, IL, USA).

\section{Statistical analysis}

Statistical analysis was performed using SPSS version 19.0 (SPSS Inc, IBM Company). A $P$-value of 0.005 was considered statistically significant. For calculation of differences, Wilcoxon test was chosen, as normal distribution was not expected.

\section{Results}

\section{Patient data baseline}

Thirty-eight patients with histopathologically proven ocular surface SCC between January 2005 and April 2012 were enrolled. According to the AJCC classification, 20 patients $(53 \%)$ had stage T3 tumours and 18 patients (47\%) had stage T4 tumours. The mean patient age was 70.6 years (33.5-92.6 years). The majority of patients $(58 \%)$ were male $(n=22)$. The mean follow-up was 24.2 months (22.3-71 months).

Thirty-two percent of the patients showed at least one of the reported risk factors in the medical history $(n=12)$. Seven patients $(18 \%)$ were smokers, five patients $(13 \%)$ were under immunosuppression, two patients $(5 \%)$ 
Table 1 American Joint Committee on cancer classification of ocular surface squamous neoplasia

\begin{tabular}{ll}
\hline Primary tumour & Tumour cannot be assessed \\
TX & Tumour absent \\
T0 & Tumour present as carcinoma in situ/conjunctival intraepithelial neoplasia \\
Tis & Tumour present with largest basal diameter $\leq 5$ mm \\
T1 & Tumour present with largest diameter $>5$ mm; no invasion of adjacent structures \\
T2 & Tumour invades adjacent structures ${ }^{\mathrm{a}}$ excluding the orbit \\
T3 & Tumour invades the orbit without further extension \\
T4 & Tumour invades orbital soft tissues, without bone invasion \\
T4a & Tumour invades bone \\
T4b & Tumour invades adjacent paranasal sinuses \\
T4c & Tumour invades brain \\
T4d & \\
& \\
Regional lymph nodes & \\
NX & Regional lymph nodes cannot be assessed \\
N0 & Regional lymph node metastasis absent \\
N1 & Regional lymph nodes present \\
& \\
Distant metastasis & \\
MO & Distant metastasis absent \\
MX & Distant metastasis present \\
\hline
\end{tabular}

adjacent structures include cornea, forniceal conjunctiva, palpebral conjunctiva, tarsal conjunctiva, intraocular compartments, caruncle, lacrimal punctum, canaliculi, semilunar fold, anterior or posterior eyelid lamellae, and/ or eyelid margin. ${ }^{23}$

suffered from atopic diseases, and one patient (3\%) had a human immunodeficiency virus infection. The tumour characteristics and demographic data are listed in Tables 2 and 3.

\section{Clinical outcome}

Thirty-five patients (92\%) had primarily surgical tumour excision upon presentation in our department. In one of the 38 patients $(3 \%)$, the tumour could not be surgically resected, as the cranium had already been invaded at first presentation. No therapeutic benefit was expected. Another patient required orbital exenteration, but was not fit enough to undergo general anaesthesia, which is necessary for this procedure. In the third patient, the surgery was not performed, as he refused to give consent for the indicated orbital exenteration.

Overall, the most frequent surgical procedure on first presentation was a local excision of SCC $(n=25$ patients, $71 \%)$. Orbital exentration was performed in 10 patients $(28 \%)$.

Of the treated cases, 20 patients $(57 \%)$ did not show a progression of the disease during follow-up and did not require any additional surgical treatment. Of the patients with primary local excision of the advanced tumour (stage T3 and T4), 13 patients (52\%) had recurrence during follow-up. Patients following orbital exenteration on first presentation had recurrence of disease in $20 \%$ $(n=2)$, one patient thereof presented with lymph node metastasis. There was no difference in recurrence in the two groups $(P=0.6)$.
However, none of the patients with primarily local excision required an orbital exenteration during followup. These recurrences were handled by further local surgical excision.

Average time to recurrence for all treated patients was 24 months in the mean (minimun 4 months, maximum 68 months, SD =22). Interestingly, $70 \%$ of recurrences presented within the first year of follow-up.

Of the patients with primary local excision, recurrence was seen 30 months in the mean (minimum 4 months, maximum 68 months, $\mathrm{SD}=27)$.

A group of patients $(37 \%, n=14)$ did not show clear tumour margins. Therefore, all of these patients also were treated with adjuvant topical IFN $\alpha-2 b$. There was no difference in rate of recurrence between the IFN $\alpha$-2b-treated vs untreated group $(P=1.0)$. There was also no statistical difference in recurrences in patients with positive or negative tumour margin after excision $(P=1.0)$.

Overall, five patients (13\%) died because of their underlying cancer; among these, three patients had not undergone surgical treatment. The other two patients showed progressive disease with intracranial involvement. They received palliative treatment with chemotherapy.

Of the patients who died due to underlying SCC, the mean time of survival was 25 months (minimum 5 months, maximum 53 months, SD $=20$ ). Patients who did not undergo surgery had a mean time of survival of only 13 months (minimum 5 months, maximum 29 months, $\mathrm{SD}=13$ ). 
Table 2 Tumour characteristics (at first presentation)

\begin{tabular}{|c|c|}
\hline Feature & Tumours, No. $(\%),(\mathrm{n}=38$ \\
\hline \multicolumn{2}{|l|}{ AJCC clinical category } \\
\hline Tis & 0 \\
\hline $\mathrm{T} 1$ & 0 \\
\hline $\mathrm{T} 2$ & 0 \\
\hline T3 & $20(53 \%)$ \\
\hline T4 & $18(47 \%)$ \\
\hline $\mathrm{T} 4 \mathrm{a}$ & $13(34 \%)$ \\
\hline $\mathrm{T} 4 \mathrm{~b}$ & $3(8 \%)$ \\
\hline $\mathrm{T} 4 \mathrm{c}$ & 0 \\
\hline $\mathrm{T} 4 \mathrm{~d}$ & $2(5 \%)$ \\
\hline No & 36 \\
\hline N1 & $2(5 \%)$ \\
\hline M0 & $37(97 \%)$ \\
\hline M1 & $1(3 \%)$ \\
\hline \multicolumn{2}{|l|}{ Tissue involved } \\
\hline Cornea & $10(26 \%)$ \\
\hline Bulbar conjunctiva & $35(92 \%)$ \\
\hline Forniceal conjunctiva & $30(79 \%)$ \\
\hline Tarsal conjunctiva & $28(74 \%)$ \\
\hline Caruncle & $8(21 \%)$ \\
\hline Lacrimal punctum & $5(13 \%)$ \\
\hline Canaliculi & 0 \\
\hline Semilunar fold & $16(42 \%)$ \\
\hline Anterior lamella & $6(16 \%)$ \\
\hline Posterior lamella & $7(18 \%)$ \\
\hline Eyelid margin & $4(11 \%)$ \\
\hline Intraocular components & $2(5 \%)$ \\
\hline Orbit & $18(47 \%)$ \\
\hline \multicolumn{2}{|l|}{ Growth pattern } \\
\hline Nodular & $7(18 \%)$ \\
\hline Diffuse & $31(82 \%)$ \\
\hline
\end{tabular}

Abbreviations: AJCC, American Joint Committee on Cancer; M, distant metastasis; $\mathrm{N}$, lymph node; $\mathrm{T}$, tumour; Tis, carcinoma in situ.

Table 3 Demographics

\begin{tabular}{lc}
\hline Characteristics & $\mathrm{n}=38$, No. $(\%)$ \\
\hline $\begin{array}{l}\text { Age in year, } \\
\text { mean (range) }\end{array}$ & 71 (minimum 33.5, \\
maximum 92.6 years) \\
Gender & \\
Male & $22(58 \%)$ \\
Female & $16(42 \%)$ \\
& \\
Risk factors & \\
Smoking & $7(18 \%)$ \\
Atopy & $2(5 \%)$ \\
HIV infection & $1(3 \%)$ \\
Immunosuppression & $5(13 \%)$ \\
\hline
\end{tabular}

The Kaplan-Meier estimation of the mean survival time revealed a mean survival of 24 months $(\mathrm{SD}=8.8$; $95 \% \mathrm{CI}=16.7-31.7)$ after initial histopathologically proven SCC.

\section{Discussion}

Although for early-stage SCC different treatment modalities including IFN $\alpha-2 b$, mitomycin $C$, and 5-fluorouracil exist, ${ }^{11}$ for advanced SCC the surgical treatment, including orbital exenteration remains the main option.

Recurrences after surgical treatment of SCC are frequent. $^{21,22}$ However, depending on the study, the rate of recurrence differs between 5 and $56 \%$, with variable follow-up times. As SCC is somewhat a rare disease, many studies on the recurrence rates of SCC examine a mixture of advanced and low-grade SCC as well as conjunctival dysplasia with variable extent of epithelial involvement. ${ }^{17,18,20,24,25}$

Kim et al ${ }^{18}$ reported a recurrence rate of $0 \%$ following the treatment of giant ocular surface neoplasia with topical and or intralesional injection with IFN $\alpha-2 b$ during a follow-up of 11 months (range, 2-43 months). Of the 18 cases observed in this study, 28\% $(n=5)$ had additional treatment with excisional surgery, cryotherapy, continuation of the treatment with topical IFN $\alpha-2 b$, and photodynamic therapy with continuation of the treatment with topical IFN $\alpha-2 b$. The rest of the patients, $72 \%(n=13)$, showed complete resolution of the tumour. However, $11 \%(n=2)$ developed new remote tumours in the tarsal and forniceal conjunctiva.

In our data of surgical excision of advanced SCC, almost $60 \%$ did not require any additional surgical treatment. Most of the patients were treated by local excision of the tumour. However, one-third of the patients required orbital exenteration on first presentation because the tumour extension was too advanced for local excision. There is a debate on the strategy of surgical management of SCC. In many cases, patients and physicians avoid the aesthetically unsatisfying surgery of orbital exenteration.

Of note, in our data none of the cases that underwent primary local excision of the advanced tumour required orbital exenteration thereafter, and all local recurrences of the tumour could be managed by only additional surgical excision.

All patients were enrolled in a strict follow-up programme with clinical visits every 3-6 months. It is noteworthy that most of the recurrences occurred during the first year of follow-up; however, 30\% occurred more than 1 year after surgical treatment. This is an aspect that we consider important, when following up patients undergoing excision of advanced-stage SCC. Follow-up visits should therefore be scheduled on a regular basis as recurrences of SCC may be found even after more than 5 years as shown in our series by a maximum follow-up of 68 months. 
Positive surgical margins after excision have been discussed to increase the risk of recurrence after excision. ${ }^{22}$ We failed to report a higher incidence of recurrences in cases that had positive margins on surgical excision of the tumour. However, in our study, patients with positive tumour margins were additionally treated with topical IFN $\alpha-2 b$. Therefore, the results of the prospective value of positive tumour margins in our study must be interpreted with caution. We also did not detect a difference in the recurrence rate of patients being treated adjuvantly with INF $\alpha-2 b$. This may also be related to the treatment of cases with positive margins only. Until now, there is no established treatment protocol upon the duration of adjuvant topical INF $\alpha-2 b$ treatment. In our study we have chosen to treat patients for 6 weeks only while other groups have reported longer duration of topical treatment. ${ }^{17,18,20}$

Although it seemed that patients with local excision of the tumour had a higher incidence of recurrences than patients who underwent orbital exenteration (52 vs 20\%), this difference was not statistically significant. Yet, given the small sample size and the low recurrence rate in the group of patients that underwent exenteration, this must be interpreted with caution.

The demography of the patients with advanced SCC is in accordance with the literature: ${ }^{11}$ most of our cases were male and the average age at presentation was 71 years. There are several risk factors known that influence the development of SCC: ultraviolet-B radiation, ionizing radiation, human papilloma virus infection, human immunodeficiency virus infection, cigarette smoke, and atopy. In our study one-third of patients showed at least one reported risk factor.

It seems that there is a proportion of patients that may not undergo the procedure of exenteration in cases of late-stage tumour. In these cases, a high mortality from the disease should be considered: all of the patients in our study who did not undergo surgery died owing to progression of the cancer after a mean time of 13 months.

Our study bears several weaknesses. First, it is a retrospective study with all its limitations. However, with regard to the low incidence of advanced SCC in general, we believe that retrospective studies are the only option to investigate a larger number of patients. In addition, there is a wide variety of surgical treatment options available. We only investigated local excision and orbital exenteration, and did not focus on other treatment options such as cryotherapy. ${ }^{6}$ This is due to the fact that, in our setting, we aimed for negative surgical tumour margins that do not require adjuvant treatment such as cryotherapy. Only in the event of histologically proven positive tumour margins, patients received additional treatment, in our case, topical IFN $\alpha-2 b$.
In conclusion, an extensive surgical approach is sometimes inevitable as shown by a high rate of primary orbital exenterations. Recurrences seem to occur more frequently after excision of advanced tumour compared with the literature for early-stage disease. Patients with surgical excision of advanced-stage disease should be reviewed closely as recurrences may occur even after more than 5 years. However, on early detection, these recurrences may be handled by local surgical excision.

\section{Summary}

What was known before

- Squamous cell carcinoma (SCC) of the conjunctiva is a rare malignancy.

- Treatment modalities vary greatly nowadays.

What this study adds

- Advanced stages of conjunctival SCC can be treated with surgical excision.

- However, an orbital exenteration is sometimes inevitable for tumour control.

- Recurrences may occur even after 5 years.

\section{Conflict of interest}

The authors declare no conflict of interest.

\section{Author Contributions}

CM has contributed to conception and design of the study, has collected data and contributed to writing and critically revision of the final manuscript. AW, AK, and $\mathrm{CH}$ have contributed to conception and design of the study and contributed to writing and critical revision of the final manuscript. AK, CD, and AG have collected data and contributed to writing and critical revision of the final manuscript. All authors have given approval for the final manuscript submitted.

\section{References}

1 Sun EC, Fears TR, Goedert JJ. Epidemiology of squamous cell conjunctival cancer. Cancer Epidemiol Biomarkers Prev 1997; 6(2): 73-77.

2 Iliff WJ, Marback R, Green WR. Invasive squamous cell carcinoma of the conjunctiva. Arch Ophthalmol 1975; 93(2): 119-122.

3 Shields CL, Shields JA. Tumors of the conjunctiva and cornea. Surv Ophthalmol 2004; 49(1): 3-24.

4 Walsh-Conway N, Conway RM. Plaque brachytherapy for the management of ocular surface malignancies with corneoscleral invasion. Clin Exp Ophthalmol 2009; 37(6): 577-583. 
5 Galor A, Karp CL, Oellers P, Kao AA, Abdelaziz A, Feuer W et al. Predictors of ocular surface squamous neoplasia recurrence after excisional surgery. Ophthalmology 2012; 119(10): 1974-1981.

6 Peksayar G, Soyturk MK, Demiryont M. Long-term results of cryotherapy on malignant epithelial tumors of the conjunctiva. Am J Ophthalmol 1989; 107(4): 337-340.

7 Lommatzsch P. Beta-ray treatment of malignant epithelial tumors of the conjunctiva. Am J Ophthalmol 1976; 81(2): 198-206.

8 Shields JA, Shields CL, De Potter P. Surgical management of conjunctival tumors. The 1994 Lynn B. McMahan Lecture. Arch Ophthalmol 1997; 115(6): 808-815.

9 Karp CL, Moore JK, Rosa Jr, RH. Treatment of conjunctival and corneal intraepithelial neoplasia with topical interferon alpha-2b. Ophthalmology 2001; 108(6): 1093-1098.

10 Spadea L, Petrucci R, Balestrazzi E. excimer laser phototherapeutic keratectomy for recurrent intraepithelial corneoconjunctival carcinoma. J Cataract Refract Surg 2002; 28(11): 2062-2064.

11 Sepulveda R, Pe'er J, Midena E, Seregard S, Dua HS, Singh AD et al. Topical chemotherapy for ocular surface squamous neoplasia: current status. Br J Ophthalmol 2010; 94(5): 532-535.

12 Chen C, Louis D, Dodd T, Muecke J. Mitomycin C as an adjunct in the treatment of localised ocular surface squamous neoplasia. Br J Ophthalmol 2004; 88(1): 17-18.

13 Hirst LW. Randomized controlled trial of topical mitomycin C for ocular surface squamous neoplasia: early resolution. Ophthalmology 2007; 114(5): 976-982.

14 Yeatts RP, Engelbrecht NE, Curry CD et al. 5-Fluorouracil for the treatment of intraepithelial neoplasia of the conjunctiva and cornea. Ophthalmology 2000; 107(12): 2190-2195.

15 Schechter BA, Schrier A, Nagler RS, Smith EF, Velasquez GE. Regression of presumed primary conjunctival and corneal intraepithelial neoplasia with topical interferon alpha- $2 \mathrm{~b}$. Cornea 2002; 21(1): 6-11.

16 Maskin SL. Regression of limbal epithelial dysplasia with topical interferon. Arch Ophthalmol 1994; 112(9): 1145-1146.

17 Shields CL, Kaliki S, Kim HJ, Al-Dahmash S, Shah SU, Lally SE et al. Interferon for ocular surface squamous neoplasia in 81 cases: outcomes based on the American Joint Committee on Cancer classification. Cornea 2013; 32(3): 248-256.

18 Kim HJ, Shields CL, Shah SU, Kaliki S, Lally SE. Giant ocular surface squamous neoplasia managed with interferon alpha- $2 \mathrm{~b}$ as immunotherapy or immunoreduction. Ophthalmology 2012; 119(5): 938-944.

19 Schechter BA, Koreishi AF, Karp CL, Feuer W. Long-term follow-up of conjunctival and corneal intraepithelial neoplasia treated with topical interferon alfa-2b. Ophthalmology 2008; 115(8): 1291-6, 6 e1.

20 Shah SU, Kaliki S, Kim HJ, Lally SE, Shields JA, Shields CL. Topical interferon alfa- $2 \mathrm{~b}$ for management of ocular surface squamous neoplasia in 23 cases: outcomes based on American Joint Committee on Cancer classification. Arch Ophthalmol 2012; 130(2): 159-164.

21 Erie JC, Campbell RJ, Liesegang TJ. Conjunctival and corneal intraepithelial and invasive neoplasia. Ophthalmology 1986; 93(2): 176-183.

22 Tabin G, Levin S, Snibson G, Loughnan M, Taylor H. Late recurrences and the necessity for long-term follow-up in corneal and conjunctival intraepithelial neoplasia. Ophthalmology 1997; 104(3): 485-492.

23 Edge SB, Compton CC. The American Joint Committee on Cancer: the 7th edition of the AJCC cancer staging manual and the future of TNM. Ann Surg Oncol 2010; 17(6): 1471-1474.

24 Karp CL, Galor A, Chhabra S, Barnes SD, Alfonso EC. Subconjunctival/perilesional recombinant interferon alpha2b for ocular surface squamous neoplasia: a 10-year review. Ophthalmology 2010; 117(12): 2241-2246.

25 Yousef YA, Finger PT. Squamous carcinoma and dysplasia of the conjunctiva and cornea: an analysis of 101 cases. Ophthalmology 2012; 119(2): 233-240.

(c) (i) (-) $\Theta$ This work is licensed under a Creative Commons Attribution-NonCommercialNoDerivs 3.0 Unported License. The images or other third party material in this article are included in the article's Creative Commons license, unless indicated otherwise in the credit line; if the material is not included under the Creative Commons license, users will need to obtain permission from the license holder to reproduce the material. To view a copy of this license, visit http:// creativecommons.org/licenses/by-nc-nd/3.0/ 